\title{
Molecular Clouds in the LMC Observed with NANTEN II. Comparison with Stellar Clusters
}

\author{
R. Yamaguchi, R. Abe, A. Hara, T. Hayakawa, S. Kato, A. Kawamura, \\ A. Mizuno, N. Mizuno, H. Ogawa, T. Onishi, H. Saito, K. Tachihara, \\ K. C. Xiao, N. Yamaguchi, and Y. Fukui \\ Department of Astrophysics, Nagoya University, Chikusa-ku, Nagoya \\ 464-8602, Japan
}

\section{Y. Yonekura}

Earth and Life Sciences, Osaka Prefecture University, Gakuencho 1-1, Sakai, Osaka 599-8531, Japan

\begin{abstract}
We have made ${ }^{12} \mathrm{CO}(J=1-0)$ observations of the LMC with the NANTEN millimeter-wave telescope and identified about 100 distinct giant molecular clouds (GMCs). A detailed comparison of the GMCs with stellar clusters and a UV image is discussed.
\end{abstract}

\section{Introduction}

The LMC possesses "populous clusters" whose properties are intermediate between the Galactic open and globular clusters (e.g., Hodge 1961). Since some of the populous clusters are younger than a few $10 \mathrm{Myr}$ (e.g., Searle, Wilkinson, \& Bagnuolo 1980; van den Bergh 1981), their parental molecular clouds may still be observable at present. Previous millimeter-wave observations of the LMC were either of low angular resolution or of limited spatial coverage, hence were not appropriate for comparisons between molecular clouds and stellar clusters. We have made a new ${ }^{12} \mathrm{CO}(J=1-0)$ survey of the LMC with the NANTEN millimeter-wave telescope of Nagoya University at the Las Campanas Observatory, Chile. The details of the observations and the results are presented by Fukui et al. (1999) and Mizuno et al. (1999). In this contribution, we present the results of a comparison between 55 newly identified GMCs and stellar clusters, and discuss the formation of clusters in the LMC.

\section{Results and Discussion}

We used 388 stellar clusters and $114 \mathrm{OB}$ associations compiled in the literature (Bica et al. 1996). They are classified into 9 types according to their ages (SWB0-VII), estimated from their color indices, $B-V$ and $U-B$.

A comparison of the GMCs with the clusters indicates that $26 \mathrm{GMCs}$, out of the 55, are associated with clusters. The youngest clusters with $\tau \leq 10$ Myr are at/near the peaks of the GMCs and often found in groups, while the older clusters (SWBI-VII) are away from the GMCs (See Fig. 1) and scattered 

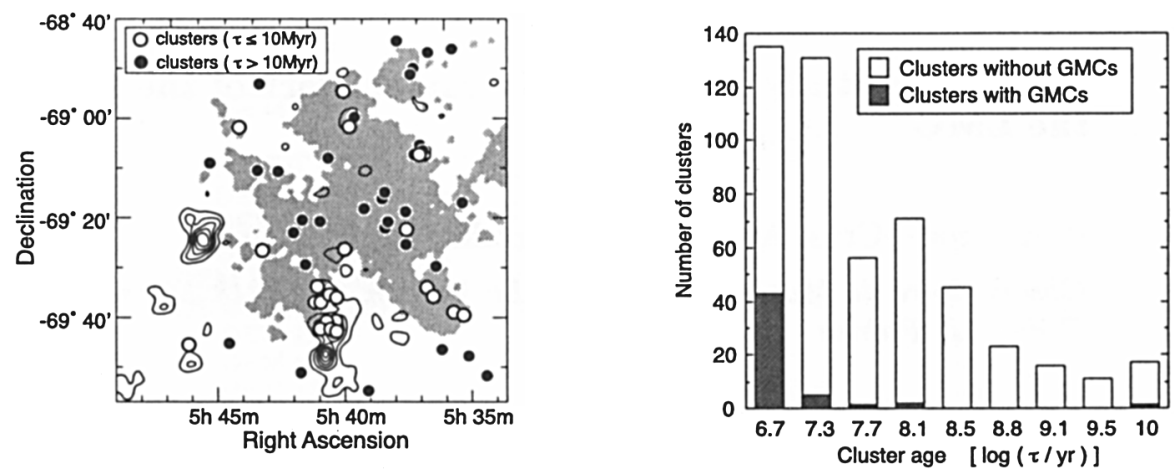

Figure 1. (left) Distribution of GMCs and clusters in the 30 Doradus region. Hatches are strong UV-emitting fields (Simith et al. 1987).

Figure 2. (right) Spatial correlation between GMCs and clusters for different ages. The ages correspond to the median of each SWB type.

spatially. Fifty-two clusters are associated with GMCs and 90\% of them are younger than $10 \mathrm{Myr}$. Fig. 2 shows the spatial correlation between the clusters and the GMCs for each age. About $30 \%$ of the youngest clusters are associated with the GMCs. The rest may be associated with smaller clouds, whose mass is below the present detection limit $\left(\mathrm{M} \sim 10^{5} \mathrm{M}_{\odot}\right)$. The older clusters (SWBIVII) show little/no correlation with the GMCs. The apparent association of the very old clusters with $\tau>30$ Myr (SWBII-VII) with GMCs may be caused by chance superpositions along the same line of sight.

Next, we compared the distribution of the youngest clusters with a $1500 \AA$ UV image (Smith et al. 1987). As shown in Fig. 1, the youngest clusters without GMCs are in strong UV radiation fields, and the GMCs tend to lie outside. Only small clouds $\left(\mathrm{M} \sim \mathrm{a}\right.$ few $\left.10^{4} \mathrm{M}_{\odot}\right)$ are in the field. These features are also found in most of the cluster forming regions, i.e., the N44 and the "Bar" regions, but not in the supergiant shells. This suggests that GMCs are rapidly dissipated and/or dissociated by UV photons from the clusters.

In summary, we suggest that a large fraction of the GMCs actively form clusters, and may be dissipated in $\leq 10 \mathrm{Myr}$ by the UV fluxes from the clusters.

Acknowledgments. We appreciate the hospitality of all the staff members of the Las Campanas Observatory of the Carnegie Institution of Washington.

\section{References}

Bica, E., et al. 1996, ApJS, 102, 57

Fukui, Y., et al. 1999, this volume

Hodge, P.W. 1961, ApJ, 133, 413

Mizuno, N., et al. 1999, this volume

Searle, L., Wilkinson, A., \& Bagnuolo, W.G. 1980, ApJ, 239, 803

Smith, A.M., Cornett, R.H., \& Hill, R.S. 1987, ApJ, 320, 609

van den Bergh, S. 1981, A\&AS, 46, 79 